\title{
Migrantes profesionistas mexicanos en Estados Unidos: Análisis de las desigualdades de género
}

\section{Educated Mexican migrants in the United States: Analysis of gender inequalities}

Recibido el 23 de abril de 2019.

Aceptado el 25 de octubre de 2019.

Publicado el 15 de noviembre de 2019.

*Autor para correspondencia: Isalia Nava Bolaños, correo electrónico, isalia@unam.mx
Esta obra está protegida bajo una Licencia Creative Commons Atribución-NoComercial 4.0 Internacional.

\author{
a Universidad Nacional Autónoma de México, Facultad de Economía, México, correo electrónico: \\ ldv@unam.mx \\ ${ }^{\mathrm{b}}$ Universidad Autónoma de Querétaro, Facultad de Contaduría y Administración, México, correo \\ electrónico: brown@unam.mx \\ c Universidad Nacional Autónoma de México, Instituto de Investigaciones Económicas, México, \\ correo electrónico: isalia@unam.mx
}

\section{Resumen:}

Este trabajo analiza comparativamente los determinantes de la integración en el mercado de trabajo estadunidense por nivel de calificación de la ocupación de los y las migrantes profesionistas mexicanos. Para cumplir con el objetivo estimamos dos modelos econométricos multinomiales que examinan las probabilidades de no ocuparse o de emplearse en puestos de distinto nivel de calificación. Con base en estos resultados se construyen escenarios. En el primero que combina el grado académico con la presencia de hijos, fue contundente la alta probabilidad para la población femenina de estar fuera de la fuerza de trabajo, con la salvedad de contar con doctorado. En el segundo escenario, en el que se relacionan las condiciones migratorias, la escolaridad y el dominio del inglés, se ilustra la vulnerabilidad como inmigrantes que se intersecta con la vulnerabilidad de género, cuando no están presentes las condiciones óptimas.

Palabras clave: migración mexicana calificada, género, ocupación, modelos multinomiales, Estados Unidos.

\section{Abstract:}

This work comparatively analyses the determinants of integration into the American labor market by qualification level of the occupation of Mexican professional migrants. To meet the objective, we estimate two multinomial econometric models that examine the odds of not being filled or used in positions of different level of qualification. Based on these results, scenarios are built. In the first that combines academic degree with the presence of children, the high probability for the fe-

CÓMO CITAR: Domínguez, L., Brown, F. y Nava, I. (2019). Migrantes profesionistas mexicanos en Estados Unidos: Análisis de las desigualdades de género. [Educated Mexican migrants in the United States: Analysis of gender inequalities]. Estudios Fronterizos, 20 , e035. doi:https://doi.org/10.21670/ref.1914035 
male population of being outside the workforce was blunt, with the exception of having a doctorate. The second scenario, which relates to immigration conditions, schooling and English proficiency, illustrates vulnerability as immigrants, when optimal conditions are not present, but in this case women have greater likely to be left out of the workforce.

Keywords: qualified Mexican migration, gender, occupation, multinomial models, United States.

\section{Introducción}

Entre las tendencias globales más sobresalientes de las últimas tres décadas está el crecimiento sin precedentes de la migración de recursos humanos educados. Así 27\% de los migrantes en el Grupo de los 20 (G20) tenía al menos un nivel de educación terciario en 2010-2011, frente al 23\% en 2000-2001, esto corresponde a 12 millones adicionales con educación terciaria, de ellos, dos tercios se encuentran en Estados Unidos, Canadá y Reino Unido (oECD, 2017). ${ }^{1}$

Al mismo tiempo, la evidencia muestra que a nivel mundial la participación de la migración femenina profesionistas es mayor en comparación con la población masculina, incluso supera a los grupos de mujeres no educadas y de hombres migrantes educados (Rodríguez, 2014). Cifras de la oECD (2017) estiman que en 2010-2011, 52\% de todos los inmigrantes altamente educados en el G20 eran mujeres, en comparación con $49 \%$ en $2000-2001$.

México es un país con una larga tradición de migración hacia Estados Unidos. En todo el mundo, en las últimas décadas se ha incrementado el porcentaje de migrantes con licenciatura o más; en 1990 pasó del 3\% a 10.2\% en 2015 (Delgado, 2015). Al mismo tiempo ha sido notable el incremento de la migración profesionista femenina. En tanto la tasa media de crecimiento anual de los hombres migrantes calificados fue de $5.9 \%$ entre 1990 y 2014 , mientras que la tasa de crecimiento promedio anual de las mujeres migrantes calificadas fue de 8.2\% (Ramírez-García y Gandini, 2016; Ramírez y Tigau, 2018). Sin embargo, a pesar de tener los mismos niveles de escolaridad, las mujeres presentan menores grados de actividad en comparación con sus congéneres varones (Gandini, 2019).

La evidencia muestra que las condiciones laborales de las migrantes mexicanas calificadas en Estados Unidos en cuanto a tasa de participación, posibilidad de empleo y ocupación de puestos en los sectores de alta calificación difieren si se comparan con las mujeres calificadas en México y con las de la generación 1.5. ${ }^{2}$ Lowell y Pederzini (2012) encuentran que la tasa de participación económica en el rango de edad que va de los 25 a los 54 años de las mujeres con maestría y doctorado en México es de $87.3 \%$ y $96.5 \%$, para las migrantes (generación 1.0$)^{3}$ estas tasas son de $72.1 \%$ y $59.5 \%$ y para la generación 1.5 son de $90.2 \%$ y $84.2 \%$, respectivamente.

\footnotetext{
${ }^{1}$ En estos países su número aumentó en 77\%, entre 2000-2001 y 2010-2011, hasta casi llegar a los 20 millones.

${ }^{2}$ La generación 1.5 se refiere "a los nacidos en la sociedad de origen, pero socializados en la sociedad de acogida" (Feixa, 2008, p. 115).

${ }^{3}$ La generación 1.0 se refiere a todas las personas nacidas en el extranjero, que reportan a México como su país de nacimiento (Lowell y Pederzini, 2012).
} 
El objetivo de esta investigación es examinar las características de la ocupación de las migrantes profesionistas mexicanas en Estados Unidos frente a los migrantes del mismo tipo para el año 2015. En particular, indagar las distintas probabilidades de ocupar empleos de alta, media y baja calificación, así como de encontrarse fuera de la fuerza de trabajo. Este análisis busca visibilizar la doble vulnerabilidad que enfrentan las mexicanas educadas en el país receptor frente a su contraparte masculina, vinculada con su situación como migrantes y como mujeres. La intención es mostrar que las desigualdades de género que permean en las esferas pública (mercado) y privada (hogar) inciden en forma diferenciada en la selección de la fuerza de trabajo y en los distintos tipos de ocupación por nivel de calificación, tal como ocurre en migrantes calificadas en otros países. En el estudio realizado por Ramírez-García y Gandini (2016) se compara la situación laboral de las mujeres mexicanas calificadas en Estados Unidos con otros grupos de mujeres migrantes o nativas. En este trabajo nos interesa examinar las desventajas de las mexicanas profesionistas frente a los hombres. En particular se busca demostrar, en primer lugar, que la carencia de ciudadanía y la deficiencia en el dominio del inglés disminuyen las oportunidades de conseguir empleo y de ocupar puestos de alta calificación, pues para las mujeres, la desigual distribución del cuidado de los hijos y su situación conyugal son circunstancias que las ponen en desventaja.

Para cumplir con el objetivo estimamos dos modelos econométricos multinomiales (uno para mujeres y otro para hombres) que examinan las probabilidades de no ocuparse o de emplearse en puestos de distinto nivel de calificación (alta, media y baja). Usamos como fuente los microdatos de la American Community Survey (ACs). Las variables explicativas se agruparon en tres grandes categorías: i) características sociodemográficas (grupos de edad, situación conyugal y presencia de hijos), ii) capital humano (nivel de escolaridad, formación académica y afluencia en el idioma inglés) y iii) condiciones migratorias (ciudadanía y tiempo de estancia en Estados Unidos).

El documento se integra por cuatro secciones más esta breve introducción y las conclusiones. En la primera sección se revisa el concepto de migración profesionista (MP); se dedicó particular atención a identificar el nivel de calificación de las ocupaciones. En la segunda sección se abordan los principales antecedentes en los estudios de migración y género y la necesidad de considerar en el análisis de MP las desigualdades entre mujeres y hombres, que dejan a las primeras en desventaja. En el tercer apartado se presentan las características sociodemográficas, de capital humano y migratorias. En la cuarta parte se especifica el modelo multinomial para estimar las probabilidades de emplearse en puestos de distinto nivel de calificación. Con base en estos resultados se construyeron dos escenarios donde se toma en cuenta $i$ ) la presencia de hijos y el grado académico y $i$ ) las condiciones migratorias, escolaridad y dominio del idioma inglés. Finalmente, presentamos las conclusiones.

\section{Clarificación de conceptos}

El estudio de la $\mathrm{MP}^{4}$ ha sido menos abordado en comparación con otros temas vinculados con este fenómeno demográfico, debido a la ausencia de información estadística; a las dificultades para establecer comparaciones en el tiempo y el espacio, y a la falta de un concepto que identifique al migrante profesionista (Gaspar y Chávez, 2016).

\footnotetext{
${ }^{4}$ En la clarificación de conceptos, seguimos de cerca el trabajo de Vázquez y Domínguez (2018).
} 
En algunos estudios se considera como MP a aquellos nacidos en el país de origen, de veinte años o más, que tienen educación terciaria (Delgado, 2015; González, 2005; Ramírez-García y Gandini, 2016). Bajo esta última definición operativa, la formación que se puede alcanzar en México con esa edad es un nivel técnico de dos o tres años. En consecuencia, esta definición incluiría a aquellos que obtuvieron su formación de licenciatura fuera de México. Ramírez-García y Gandini (2016) consideran a la MP como aquellas personas con nivel terciario (o de licenciatura) finalizado o más (posgrado).

En este trabajo los pasos seguidos para identificar a la MP de mexicanos en Estados Unidos fueron los siguientes: se consideró a aquellos individuos que llegaron a Estados Unidos desde los 25 años $^{5}$ y que al menos contaban con licenciatura (o equivalente) antes de emigrar (Batalova, Fix y Creticos, 2008). La definición de las ocupaciones según nivel de calificación se realizó a partir del porcentaje de empleados con al menos licenciatura (Vázquez y Domínguez, 2018). Esta forma de agrupar las ocupaciones coincide con la Clasificación Internacional Uniforme de Ocupaciones (cIUO, 2008) de la Organización Internacional del Trabajo. Se identificaron tres niveles de calificación: alta, media y baja.

Las ocupaciones de alta calificación corresponden a aquellas donde al menos $60 \%$ del total de los trabajadores tienen licenciatura. Ejemplos de ocupaciones en este nivel son los científicos, intelectuales y profesionistas, gerentes de ventas y comercialización; ingenieros civiles; profesores de enseñanza secundaria; médicos; músicos; personal de enfermería asistentes en el quirófano, y analistas de sistemas informáticos. Estas ocupaciones involucran tareas que exigen la solución de problemas complejos, la toma de decisiones y la creatividad sobre la base de un amplio conocimiento teórico y fáctico en una especialización determinada.

Para las ocupaciones de calificación media se consideró a aquellas que tienen un porcentaje de empleados con al menos licenciatura, de entre $20 \%$ y $59 \%$. Ejemplos de estas ocupaciones son: supervisores en la industria, técnicos de laboratorios médicos, secretarios jurídicos, técnicos de soporte informático, entre otros. Estas abarcan el desempeño de tareas técnicas y prácticas complejas que exigen un conjunto de conocimientos técnicos y de procedimientos en un área especializada.

Las ocupaciones de calificación baja corresponden a aquellas en donde el porcentaje de trabajadores con nivel de licenciatura es menor a 20\%. Algunos ejemplos son operadores o encargados de mantenimiento de máquinas, sastres o modistos, conductores de autobuses, vendedores de tiendas, meseros, electricistas, hasta aquellas que implican tareas físicas o manuales, simples y rutinarias como peones de carga, jardinería, ayudantes de cocina, entre otros. Comprenden las tareas de más bajas competencias y abarcan desde aquellas en donde se requiere habilidad para leer instrucciones o hacer operaciones aritméticas simples.

\section{Migración y género}

Los movimientos poblacionales están relacionados con el género dado que las razones por las cuales migran hombres y mujeres son diferentes, así como sus canales para

\footnotetext{
${ }^{5}$ En la encuesta hay una pregunta que se refiere a la edad actual y otra, a los años de residencia. De esta manera pudimos determinar la edad en la cual llegaron a Estados Unidos.
} 
migrar y sus experiencias (International Organization for Migration [IOM], 2010). A inicio de la década de los ochenta los hombres emigraban a Estados Unidos, mientras que las mujeres permanecían como responsables en el hogar de origen o emigraban como acompañantes en carácter de esposas, hijas o madres del migrante varón (Cárdenas, 1983). Sin embargo, con el paso del tiempo esta figura se ha transformado, incluso algunas de ellas emigran por su cuenta en busca de mejores oportunidades económicas, sobre todo, quienes tienen altos niveles de escolaridad (Kanaiaupuni, 2000). Por lo tanto, la migración femenina no necesariamente involucra un patrón de proveedor masculino, como se verá más adelante (IOM, 2010). La investigación de la migración con lentes de género se ha centrado en visibilizar la doble vulnerabilidad que enfrentan las mujeres en el país receptor: como migrantes y como mujeres. Ambas son particularmente importantes para las migrantes profesionistas, tema que nos ocupa en este trabajo. Sin embargo, como señala Gandini (2019) los estudios sobre migración calificada están alejados del enfoque de género.

El contexto social en el que ocurre la migración está influido por las desigualdades según sexo y las relaciones familiares, lo cual plantea la necesidad de analizar este fenómeno. Por ejemplo, Kanaiaupuni (2000) y Cerrutti y Massey (2004) encontraron roles diferenciados entre mujeres y hombres; Meares (2010), a su vez, identifica un impacto negativo en la profesión de las mujeres acompañado por el incremento en las responsabilidades del hogar. Cabe señalar que las migrantes calificadas también enfrentan, en mayor medida que los hombres, problemas de descualificación y subutilización, en donde la experiencia laboral obtenida en el país de origen se desperdicia, al desempeñarse en trabajos distintos a los de su formación educativa (Ramírez-García y Gandini, 2016). En otros, puede implicar que aun teniendo credenciales académicas, las mujeres deban permanecer fuera de la fuerza de trabajo por periodos importantes (Gottfried, 2013).

Entre la bibliografía que analiza el tema de migración y género se identifica un grupo de autores que examinan los factores que inciden en la decisión de migrar de unos y otras, con algunas diferencias, como se señala a continuación.

La población de migrantes mexicanos a Estados Unidos se caracteriza por una selección positiva con respecto a la educación, característica que se ha incrementado en las últimas décadas (Feliciano, 2008). Cabe mencionar que esta selectividad es más alta entre las migrantes que en sus contrapartes masculinos, lo cual es confirmado por Kanaiaupuni (2000).

Un aspecto de gran interés es la relación entre la migración femenina, el trabajo doméstico remunerado y el cuidado de la familia, en las cadenas globales de cuidado. Es un hecho que en países desarrollados como Estados Unidos se está produciendo un déficit de los cuidados (Gottfried, 2013). Por un lado, en estos países el modelo previo de organización social de los cuidados, que se caracterizaba por la división sexual del trabajo y la escisión social de espacios público/privado, está quebrando, y una de las vías de respuesta ha sido externalizar parte de las tareas que antes se realizaban en los hogares, o mercantilizar parte del trabajo doméstico y de cuidados no remunerado (Orozco, 2007). Así, algunas migrantes se emplean como nanas o cuidadoras de personas mayores, muchas veces luego de dejar a sus hijos atrás en su país de origen. Por otro lado, y más pertinente para la migración educada, es la insuficiente oferta de enfermeras y cuidadoras de enfermos. De ahí que los distintos 
gobiernos hayan facilitado el acceso migratorio a enfermeras y personas capacitadas en labores similares. ${ }^{6}$

Cabe mencionar que aún prevalece el patrón de una mayor proporción de mujeres educadas que trabajan con horarios parciales, así como una alta segregación de género hacia trabajos que involucran menos complejidad y autonomía, principalmente en los sectores de alta tecnología (Benería y Roldán, 1987). Además, la recompensa salarial de las mujeres profesionistas es deficitaria (Gandini, 2019).

En relación con la vulnerabilidad como migrantes, las mujeres se enfrentan al escaso dominio del inglés, la falta de fluidez y el marcado acento, así como a la insuficiencia de redes de trabajo (Zhou, 2003). Así, en Estados Unidos, la tasa de empleo de las mujeres del tercer mundo en edad de trabajo (25-54) en 2010 fue casi $20 \%$ más baja que el promedio de la tasa de empleo para todas las mujeres en ese grupo de edad (Eurostat, 2010). Por otra parte, en la medida en que entren como acompañantes de su esposo, la situación migratoria puede desfavorecer su incorporación en el mercado laboral.

Con respecto a la vulnerabilidad de género, las migrantes calificadas tienen distintos patrones de empleabilidad (Man, 2004; Meares, 2010). La evidencia empírica en Estados Unidos muestra que mujeres con educación universitaria pueden ocupar empleos que no demandan esos conocimientos. La razón la hallamos en que, a pesar del enorme cambio cultural ocurrido en muchos países - gracias al cual las mujeres han logrado obtener grados universitarios y posgrados, así como una más amplia participación en el mercado laboral, además de nuevas leyes y modificaciones normativas en pro de la igualdad entre mujeres y hombres en distintos ámbitos-, es todavía un hecho para el caso norteamericano que la mujer tiene una responsabilidad desproporcionada en el cuidado de los hijos y del hogar (Gottfried, 2013). Lo cual dificulta su competencia en igualdad de términos con los hombres fuera de la casa.

Se ha reconocido que hay normas informales y culturales que determinan la segregación de género en ciertos sectores de la economía y en modalidades de trabajo, así como la segregación vertical (Stone, 1994). Igualmente, las construcciones socioculturales de los roles y estereotipos de género establecen quién es la autoridad en la toma de decisiones o la propiedad de los activos económicos (Elson, 1999). ${ }^{7}$ De ahí que algunas mujeres consideran que pueden tener mayor retribución para su capital humano en Estados Unidos, sin embargo, es posible que se encuentren con un mercado altamente segregado y que, por tanto, no lo logren (IOM, 2010).

Una explicación es que la presencia de hijos remite a responsabilidades familiares que tienden a afectar más a las mujeres que a los hombres. La relativa falta de infraestructura de apoyo en Estados Unidos para los padres trabajadores obliga a las familias a encargarse de las tareas de cuidado, ya sea mediante el trabajo no remunerado de algún miembro de la familia o contratando el servicio en el mercado (Gottfried, 2013). ${ }^{8}$ A ello se suma la importación de los patrones culturales de género

\footnotetext{
${ }^{6}$ Según un estudio de la IOM-OECD (2014), los sistemas de admisión tienden a favorecer a los hombres en profesiones como gerencia de negocios, tecnologías de información e ingeniería y a las mujeres como enfermeras, profesoras y trabajadoras sociales.

${ }^{7}$ Para una discusión más amplia véase Perrons (2015).

${ }^{8}$ Esto es así porque Estados Unidos es uno de los dos países en el mundo que no cuenta con licencia de maternidad pagada. Algunas empresas privadas la dan, pero su cobertura es baja. Por otra parte, Estados Unidos es uno de los países de la OCDE con menor gasto público en educación y cuidado en la primera infancia como porcentaje del PIB con $0.5 \%$ por debajo del promedio $(0.7 \%)$ y muy por debajo de Francia, Nueva Zelanda y los países nórdicos (OECD, 2016).
} 
predominantes en México, en donde los roles atribuidos a los hombres y las mujeres casados afectan las decisiones que se toman en los países de destino. Al mismo tiempo, la familia patriarcal tradicional restringe con frecuencia la inserción de las mujeres en el trabajo y las confina a las tareas de trabajo doméstico y de cuidados no remunerado.

Habiendo señalado cada una de las vulnerabilidades entre las mujeres, cabe mencionar que ambas se intersectan, lo cual aumenta la dificultad de elegir entre trabajar o no, y sobre qué tipo de labor desempeñar (Zhou, 2003).

Con base en estos antecedentes es importante preguntarse acerca de la situación laboral de las migrantes mexicanas de alta calificación: ¿qué diferencias hay frente a la posición de los MP?

\section{Migración profesionista e inserción ocupacional}

En párrafos anteriores se enfatizó la importancia de examinar el tema de la migración de profesionistas mexicanos, así como la inserción laboral con lentes de género. A continuación detallamos algunas condiciones que inciden en la inserción de la MP según niveles de calificación de la ocupación.

La información que se presenta proviene de los microdatos de la ACs 2015, elaborada por la Oficina del Censo de Estados Unidos. Por su diseño y tamaño de la muestra la ACS es una encuesta en hogares ampliamente utilizada para el estudio de la migración calificada.

A partir de la ACs se estima que en 2015 el número (stock) de inmigrantes mexicanos con al menos licenciatura en Estados Unidos fue de 132604 hombres y 132013 mujeres. ${ }^{9}$ En la desagregación según ocupación por grado de calificación se observa que, para el caso de las mujeres, $30 \%$ de ellas se encuentran en ocupaciones de alta calificación, 9\% menos que los hombres. En las ocupaciones de calificación media no se aprecian diferencias importantes. En las ocupaciones de baja calificación son los hombres quienes registran porcentajes más altos, $34 \%$ en comparación con $25 \%$ de las mujeres, mientras que solo $9 \%$ de los hombres están fuera de la fuerza de trabajo y $30 \%$ de las mujeres están en esta situación, es decir, más de tres veces el número de los hombres (Figura 1).

En el análisis de la inserción laboral planteamos como variables explicativas el dominio del inglés, las condiciones migratorias y los años de residencia para analizar la vulnerabilidad como migrantes, así como la vulnerabilidad de género, la presencia de hijos y el estar casadas. Hay otras variables que pueden influir en el tipo de inserción laboral que también deben considerarse, como la edad, debido a que esta puede afectar la inserción laboral en forma distinta que los hombres. En la Tabla 1 se presentan las variables asociadas a la vulnerabilidad de género y se puede observar que, en cuanto a la distribución de hombres y mujeres en los grupos de edad, no se vislumbra un patrón muy diferenciado en las ocupaciones de alta calificación o media calificación. No obstante, entre la población fuera de la fuerza de trabajo se aprecia que el porcentaje de hombres de más de 45 años es 30 puntos porcentuales mayor que

${ }^{9}$ Del total, $71 \%$ están casados, $55 \%$ tienen hijos menores de sesis años, $65 \%$ no tienen la ciudadanía y $72 \%$ tienen dominio del inglés. 
el de las mujeres ( $86 \%$ vs. $56 \%$ ), lo que puede indicar que estar fuera de la fuerza de trabajo no es una opción para ellos en las edades más jóvenes.

Figura 1: Distribución porcentual de la MP según grado de calificación, 2015

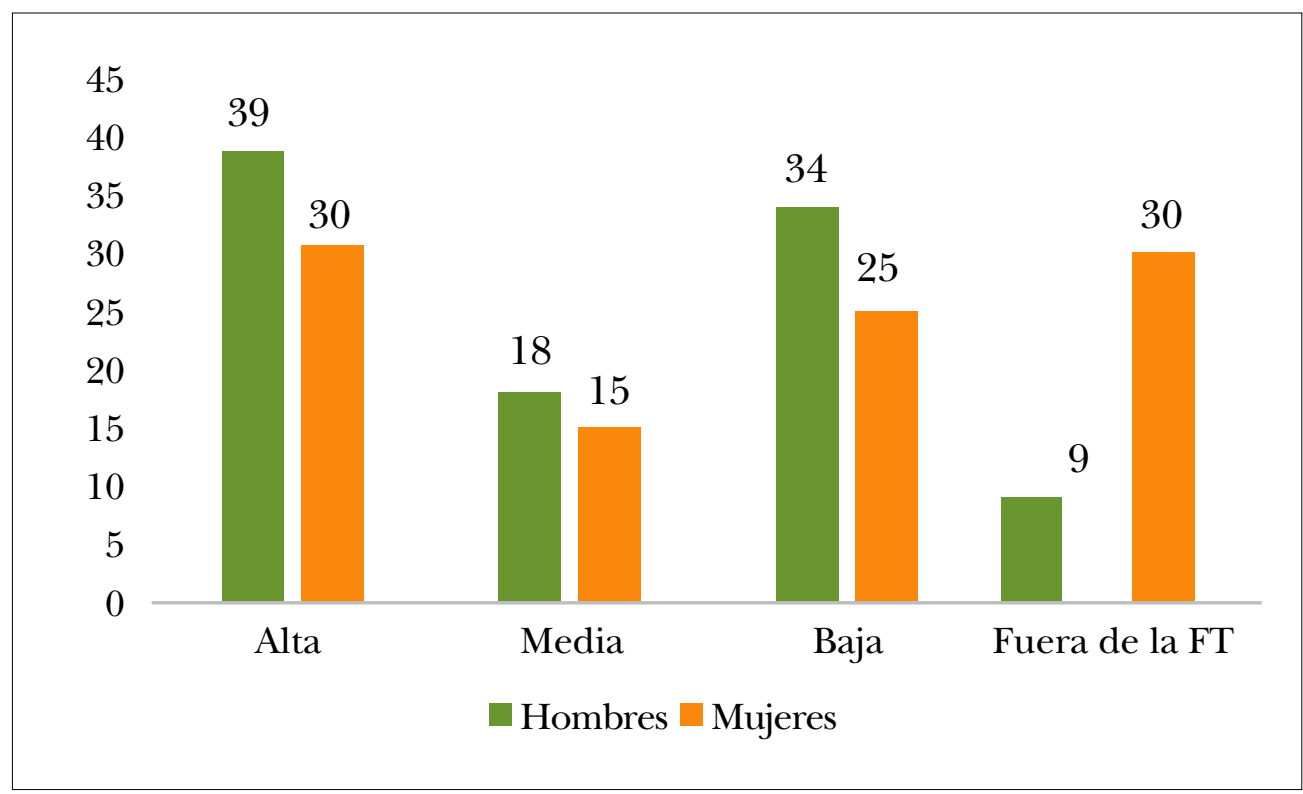

Fuente: Elaboración propia con base en Acs (2015).

En relación con la situación conyugal, el estado predominante es la unión matrimonial con algunas diferencias según grado de calificación. Por ejemplo, hay un porcentaje más alto de mujeres no casadas en las ocupaciones de calificación alta, en comparación con los varones (31\% y 24\%, respectivamente). En contraste, cuando están fuera de la fuerza de trabajo hay una mayor proporción de mujeres casadas que de hombres (75\% vs. $68 \%$ ). La composición de los hogares de mP incluye el hogar tradicional de parejas unidas por matrimonio $(71 \%)$. Pero también de hogares donde el cónyuge está ausente y con importantes diferencias por sexo: $12 \%$ de esposas ausentes, frente a 19\% de esposos. Es decir, el porcentaje de mujeres solas con responsabilidades en un hogar está por encima siete puntos del de hombres solos. Así mismo, hay hogares no familiares de hombres y mujeres; en estos casos el porcentaje de los hombres es mayor: $17 \%$ frente a 10\%, respectivamente (Tabla 1). Esto confirma que las mujeres no solo migran por motivos de reunificación familiar, sino que también son agentes independientes o proveedoras como lo señala la IOM (2010).

Además, en la Tabla 1 se aprecia la relación negativa de los hijos en el empleo de las migrantes femeninas: condición que disminuye su oportunidad de insertarse en ocupaciones de alta calificación (entre las mujeres en ocupaciones de alta calificación solo $14 \%$ tienen hijos menores de seis años, frente a $20 \%$ de los hombres en calificaciones altas) e incrementa el porcentaje fuera de la fuerza de trabajo (entre la población femenina fuera de la fuerza de trabajo, $26 \%$ tiene hijos menores de 6 años y solo $6 \%$ de los hombres fuera de la fuerza de trabajo lo está). Estos resultados coinciden con lo señalado por Caicedo (2012) y Martínez (2008) para la población migrante, y con García y Pacheco (2000) y Domínguez y Brown (2013) en la población en general. 
Tabla 1: Características sociodemográficas. Distribución porcentual de la MP, 2015

\begin{tabular}{|c|c|c|c|c|c|c|c|c|}
\hline \multirow{2}{*}{ Características } & \multicolumn{2}{|c|}{ Calificación alta } & \multicolumn{2}{|c|}{$\begin{array}{l}\text { Calificación } \\
\text { media }\end{array}$} & \multicolumn{2}{|c|}{ Calificación baja } & \multicolumn{2}{|c|}{$\begin{array}{c}\text { Fuera de la fuerza } \\
\text { de trabajo }\end{array}$} \\
\hline & $\mathrm{H}$ & M & $\mathrm{H}$ & M & $\mathrm{H}$ & M & $\mathrm{H}$ & M \\
\hline \multicolumn{9}{|c|}{ Grupos de edad } \\
\hline 25 a 35 años & 13.5 & 13.4 & 12.0 & 12.2 & 9.7 & 9.8 & 5.8 & 11.0 \\
\hline 36 a 45 años & 36.5 & 35.1 & 32.1 & 38.8 & 27.9 & 30.7 & 7.8 & 32.6 \\
\hline Más de 45 años & 50.0 & 51.6 & 56.0 & 48.9 & 62.4 & 59.5 & 86.4 & 56.4 \\
\hline \multicolumn{9}{|c|}{ Situación conyugal } \\
\hline No casado & 23.8 & 31.2 & 19.1 & 24.5 & 29.6 & 33.3 & 32.0 & 24.5 \\
\hline Casado & 76.2 & 68.8 & 80.9 & 75.5 & 70.4 & 66.7 & 68.0 & 75.5 \\
\hline \multicolumn{9}{|c|}{ Presencia de hijos } \\
\hline $\begin{array}{l}\text { Sin hijos menores de } \\
6 \text { años }\end{array}$ & 80.2 & 86.4 & 81.1 & 84.6 & 87.2 & 84.7 & 93.9 & 74.4 \\
\hline $\begin{array}{c}\text { Con hijos menores de } \\
6 \text { años }\end{array}$ & 19.8 & 13.6 & 18.9 & 15.4 & 12.8 & 15.3 & 6.1 & 25.6 \\
\hline
\end{tabular}

Nota: Los totales pueden no sumar 100 por el redondeo de cifras. Fuente: Elaboración propia con base en Acs (2015).

Por su parte, el estatus migratorio es un elemento que otorga certidumbre y estabilidad para lograr condiciones labores adecuadas, sin embargo, la Acs no proporciona información sobre la gama de visas, solo provee datos sobre la condición de ciudadanía. En cuanto a las características migratorias que se describen en la Tabla 2, se observa que, entre mayor estabilidad tienen los migrantes en cuanto a condición migratoria (ciudadanía) más adecuadas son las condiciones laborales: $47 \%$ del total de mujeres en ocupación de alta calificación frente a $38 \%$ de los hombres. Lo contrario sucede cuando están fuera del mercado de trabajo: $71 \%$ de las mujeres no tienen ciudadanía frente a $52 \%$ de los hombres.

Aproximadamente, dos tercios de la población migrante tienen más de 10 años de residencia en todos los niveles de ocupación. El porcentaje de los hombres de alta calificación con residencia entre 6 a 10 años (17\%) es mayor al de las mujeres (13\%), por el contrario, el porcentaje de ellas con residencia de 11 y más años es de $61 \%$ vs. $57 \%$ de ellos (Tabla 2).

La formación profesional sin duda ejerce una influencia importante en la inserción laboral. Se observa en la Tabla 3 que en términos generales la escolaridady, en particular, los grados académicos más altos se relacionan con un ingreso más elevado y mejores condiciones en el empleo (Walby, Gottfried, Gottschall y Osawa, 2016). En el caso de las mujeres, la educación ha probado ser de gran importancia para explicar su participación en el mercado de trabajo (Delgado, Chávez y Rodríguez, 2016; Docquier, Lowell y Marfouk, 2009). En forma clara se aprecia que el porcentaje de migrantes con nivel de licenciatura es mayor que con posgrado, tanto para hombres y mujeres. En las ocupaciones de alta calificación hay un mayor porcentaje de mujeres con licenciatura ( $59 \%$ vs. $52 \%$ ) y menor con posgrado. En contraste, en la categoría fuera de la fuerza de trabajo hay un menor porcentaje de mujeres con posgrado que de hombres $(22 \%$ vs. $36 \%$ ), aunque, en términos absolutos, el número de hombres es muy bajo. 
Tabla 2: Condiciones migratorias. Distribución porcentual de la MP, 2015

\begin{tabular}{|c|c|c|c|c|c|c|c|c|}
\hline \multirow[t]{2}{*}{ Características } & \multicolumn{2}{|c|}{$\begin{array}{l}\text { Calificación } \\
\text { alta }\end{array}$} & \multicolumn{2}{|c|}{$\begin{array}{c}\text { Calificación } \\
\text { media }\end{array}$} & \multicolumn{2}{|c|}{$\begin{array}{c}\text { Calificación } \\
\text { baja }\end{array}$} & \multicolumn{2}{|c|}{$\begin{array}{c}\text { Fuera de la fuerza de } \\
\text { trabajo }\end{array}$} \\
\hline & $\mathrm{H}$ & M & $\mathrm{H}$ & M & $\mathrm{H}$ & M & $\mathrm{H}$ & M \\
\hline \multicolumn{9}{|c|}{ Ciudadanía } \\
\hline No & 62 & 53 & 65 & 51 & 73 & 69 & 52 & 71 \\
\hline Sí & 38 & 47 & 35 & 49 & 27 & 31 & 48 & 29 \\
\hline \multicolumn{9}{|c|}{ Tiempo de residencia } \\
\hline Hasta cinco años & 26 & 26 & 25 & 24 & 17 & 14 & 10 & 19 \\
\hline 6 a 10 años & 17 & 13 & 15 & 11 & 14 & 16 & 10 & 18 \\
\hline 11 y más años & 57 & 61 & 59 & 65 & 70 & 70 & 79 & 63 \\
\hline
\end{tabular}

Nota: Los totales pueden no sumar 100 por el redondeo de cifras. Fuente: Elaboración propia con base en Acs (2015).

En relación con la especialidad de los grados académicos hay importantes diferencias (Tabla 3). En el caso de los hombres predomina la ingeniería, ciencias, matemáticas y ciencias de la computación (ICMCC) (52\% de frente a $18 \%$ de las mujeres). Por su parte, $28 \%$ del total de las mujeres se especializaron en negocios frente a $21 \%$ de los hombres; le siguen ciencias sociales y educación (17\%).

Una variable que está ligada con la formación o capacitación es el dominio del idioma inglés. La población de migrantes profesionales mexicanos se distingue por tener una posición desventajosa frente a otros grupos de migrantes (Vázquez y Domínguez, 2018). En las ocupaciones de calificación alta se observa un mayor porcentaje de hombres (91\%) con dominio del inglés frente a las mujeres (86\%). Cuando están fuera de la fuerza de trabajo $45 \%$ de las mujeres no tienen dominio del inglés frente a $30 \%$ de los hombres (Tabla 3 ).

Además de las marcadas desigualdades arriba señaladas es interesante mencionar que, como se observa en la Tabla 4, al interior del grupo de migrantes calificados existe segregación horizontal, que se manifiesta en una mayor concentración de las mujeres en ocupaciones identificadas como femeninas de acuerdo con la división sexual del trabajo (Rodríguez, 2012). En las ocupaciones de calificación alta, los varones sobresalen en ICMCC con una participación de $24 \%$ frente a $9 \%$ de ellas. Por su parte, la participación de las migrantes destaca en ocupaciones relacionadas con educación (33\% frente a $11 \%$ de ellos) y medicina ( $8 \%$ frente a $5 \%$ ). En finanzas, negocios y abogacía no hay diferencias importantes. En ocupaciones de calificación media la participación de las migrantes en servicios comunitarios y personales (22\%) y labores de oficina (32\%) sobresale frente a los migrantes, con $9 \%$ y $11 \%$, respectivamente. Por su parte, la participación de los hombres en ventas excede en 15 puntos porcentuales a la de ellas (34\% frente a $19 \%)$. 
Tabla 3: Capital humano. Distribución porcentual, 2015

\begin{tabular}{|c|c|c|c|c|c|c|c|c|}
\hline \multirow[t]{2}{*}{ Características } & \multicolumn{2}{|c|}{ Calificación alta } & \multicolumn{2}{|c|}{ Calificación media } & \multicolumn{2}{|c|}{ Calificación baja } & \multicolumn{2}{|c|}{$\begin{array}{c}\text { Fuera de la } \\
\text { fuerza de } \\
\text { trabajo }\end{array}$} \\
\hline & $\mathrm{H}$ & M & $\mathrm{H}$ & M & $\mathrm{H}$ & M & $\mathrm{H}$ & M \\
\hline \multicolumn{9}{|c|}{ Escolaridad } \\
\hline Licenciatura & 52 & 59 & 73 & 79 & 82 & 82 & 64 & 78 \\
\hline Posgrado & 48 & 41 & 27 & 21 & 18 & 18 & 36 & 22 \\
\hline \multicolumn{9}{|c|}{ Especialidad } \\
\hline Negocios & 21 & 28 & 25 & 43 & 25 & 34 & 20 & 28 \\
\hline $\begin{array}{l}\text { Ciencias, ingeniería, } \\
\text { matemáticas y ciencias } \\
\text { de la computación }\end{array}$ & 52 & 18 & 56 & 18 & 48 & 13 & 43 & 15 \\
\hline Ciencias sociales & 11 & 17 & 8 & 15 & 10 & 16 & 13 & 14 \\
\hline $\begin{array}{l}\text { Otros (educación, } \\
\text { ciencias de la salud, } \\
\text { humanidades y arte) }\end{array}$ & 16 & 36 & 11 & 24 & 17 & 37 & 23 & 42 \\
\hline \multicolumn{9}{|c|}{ Idioma inglés } \\
\hline Sí & 91 & 86 & 81 & 86 & 62 & 57 & 70 & 55 \\
\hline No & 9 & 14 & 19 & 14 & 38 & 43 & 30 & 45 \\
\hline
\end{tabular}

Nota: Los totales pueden no sumar 100 por el redondeo de cifras.

Fuente: Elaboración propia con base en Acs (2015).

También está presente la segregación vertical. Dicha segregación da cuenta de una mayor aglutinación de las mujeres en puestos de menor jerarquía, a pesar de contar con niveles de calificación similares a los de sus pares varones (Rodríguez, 2012). Así, en ocupaciones de gerencia las migrantes calificadas tienen una menor presencia, once puntos menos, tanto en ocupaciones de calificación alta como media.

Tabla 4: Distribución porcentual de la MP según ocupaciones, 2015

\begin{tabular}{|c|c|c|c|c|}
\hline \multirow{2}{*}{ Ocupaciones } & \multicolumn{2}{|c|}{ Calificación alta } & \multicolumn{2}{|c|}{ Calificación media } \\
\hline & $\mathrm{H}$ & M & $\mathrm{H}$ & M \\
\hline Gerente & 37 & 26 & 30 & 18 \\
\hline FIN-NEG & 12 & 11 & 4 & 3 \\
\hline ICMCC & 24 & 9 & & \\
\hline Educación & 11 & 33 & & \\
\hline Medicina & 5 & 8 & & \\
\hline Servicios comunitarios y personales & 5 & 7 & 9 & 22 \\
\hline Entretenimiento & 7 & 6 & & \\
\hline Oficina & & & 11 & 32 \\
\hline Ventas & & & 34 & 19 \\
\hline Otros & & & 10 & 6 \\
\hline
\end{tabular}

Nota: Los totales pueden no sumar 100 por el redondeo de cifras.

Fuente: Elaboración propia con base en Acs (2015). 
Los patrones de segregación se extienden al lugar de empleo, los migrantes calificados tienden a distribuirse en mayor proporción que sus congéneres mujeres en empresas con fines de lucro (incluye a universidades privadas) y autoempleo, este último es particularmente importante para ellos en las ocupaciones de calificación media. Las migrantes educadas tienen mayor presencia que los migrantes en sociedades sin fines de lucro y gobierno (federal, estatal o local), con más altas participaciones, en particular, en las ocupaciones de alta calificación para ellas (29\% frente a $14 \%$ ) y calificación media (18\% frente a $7 \%$ ). En las ocupaciones de baja calificación destaca $80 \%$ de los migrantes calificados frente a $65 \%$ de las migrantes que trabajan en empresa privada y las migrantes en autoempleo no incorporado, esto último está ligado a ocupaciones de servicios de comida (Tabla 5 ).

Tabla 5: Distribución porcentual de la MP según lugar de empleo, 2015

\begin{tabular}{ccccccc}
\hline & \multicolumn{2}{c}{ Calificación alta } & \multicolumn{2}{c}{ Calificación media } & \multicolumn{2}{c}{ Calificación baja } \\
\hline Lugar de empleo & H & M & H & M & H & M \\
\hline Empresa privada & 63 & 47 & 68 & 64 & 80 & 65 \\
\hline Sociedad no lucrativa & 10 & 14 & 1 & 4 & 2 & 6 \\
Gobiernos & 14 & 29 & 7 & 18 & 5 & 9 \\
Autoempleo & 13 & 9 & 23 & 13 & 12 & 20 \\
\hline
\end{tabular}

Nota: Los totales pueden no sumar 100 por el redondeo de cifras.

Fuente: Elaboración propia con base en Acs (2015).

\section{La inserción de la migración calificada en las distintas ocupaciones: un modelo multinominal}

\section{Aspectos metodológicos}

El objetivo del modelo es examinar las diferencias entre mujeres y hombres en distintos nichos del mercado en las probabilidades de ocuparse, por nivel de calificación de las ocupaciones: alta, media, baja y fuera de la fuerza de trabajo. La variable dependiente es categórica, se le asignó el número "1" a los empleados en ocupaciones en las que se demandan altas calificaciones; el "2", a las calificaciones medias; "3", a las bajas, y "4", a las personas que no tienen un puesto de trabajo.

A partir de la revisión de la literatura y del análisis descriptivo, las variables explicativas se agruparon en las siguientes tres categorías. La descripción y operacionalización de las variables se presentan en la Tabla 6.

Se espera un comportamiento no lineal para la edad; en los grupos de edades más altos, las posibilidades de empleo son mayores. En cuanto a la situación conyugal y la presencia de hijos se esperará una relación negativa con respecto a los segmentos de alta calificación para las mujeres. 
Tabla 6: Descripción de variables

\begin{tabular}{|c|c|c|}
\hline $\begin{array}{l}\text { Características } \\
\text { sociodemográficas }\end{array}$ & $\begin{array}{l}\text { Capital } \\
\text { humano }\end{array}$ & $\begin{array}{l}\text { Condiciones } \\
\text { migratorias }\end{array}$ \\
\hline $\begin{array}{l}\text { Grupos de edad } \\
1=25 \text { a } 35 \text { años } \\
2=36 \text { a } 45 \text { años } \\
3=46 \text { y más }\end{array}$ & $\begin{array}{l}\text { Nivel de escolaridad } \\
1=\text { Licenciatura } \\
2=\text { Posgrado }\end{array}$ & $\begin{array}{l}\text { Ciudadano americano } \\
1=\text { Sí } \\
0=\text { No }\end{array}$ \\
\hline $\begin{array}{l}\text { Situación conyugal } \\
1=\text { casado o unido a una pareja } \\
0=\text { viudo, divorciado, separado y } \\
\text { nunca casado }\end{array}$ & $\begin{array}{l}\text { Formación académica } \\
1 \text { = Negocios } \\
2 \text { = Ciencias, ingeniería, } \\
\text { matemáticas y ciencias de la } \\
\text { computación } \\
3=\text { Ciencias sociales } \\
\text { 4= Otros (educación, ciencias } \\
\text { de la salud, humanidades y arte) }\end{array}$ & $\begin{array}{l}\text { Tiempo de estancia en } \\
\text { Estados Unidos } \\
\text { edad-año de llegada }\end{array}$ \\
\hline $\begin{array}{l}\text { Presencia de hijos } \\
1=\text { Tiene hijos de hasta } 6 \text { años }\end{array}$ & Dominio del idioma inglés & \\
\hline $\begin{array}{l}0=\text { No tiene hijos de hasta } 6 \\
\text { años }\end{array}$ & $0=\mathrm{No}$ & \\
\hline
\end{tabular}

Fuente: Elaboración propia con base en Acs (2015).

En las características relacionadas con la situación migratoria, específicamente, con la ciudadanía, esperaríamos mayores posibilidades de estar empleado al menos en los niveles de alta y media calificación, pero también la expectativa es que haya diferencias por sexo. En lo que toca a los años de residencia, en la medida en que implican acumulación de experiencia laboral y de vida, supondríamos que más años elevan la probabilidad de empleo en las ocupaciones de alta y media calificación. También, se espera que el dominio del inglés tenga gran influencia en la integración laboral, por tanto, nuestra expectativa es que ejerza un alto impacto en la posibilidad de ser empleado en los niveles de alta y media calificación, tanto para ellas como ellos.

Las posibilidades de ubicarse en ocupaciones del nivel más alto esperamos estén asociadas con el grado académico. Es decir, ante la posesión de posgrado nuestra expectativa es que se refleje positivamente frente a tener solo licenciatura, al menos en el nivel alto. Esperaríamos que el efecto de las áreas de especialización sea distinto por sexo: mayor probabilidad de que los hombres con especialización en ICMCC se ubiquen en puestos de alta calificación, en tanto que las mujeres participan en estos puestos cuando su especialización es el área de negocios. 


\section{Probabilidad de inserción en distintas ocupaciones: Resultados}

El modelo Multinomial Logit que se estimó parte de la idea central de que no existe un orden jerárquico entre las cuatro alternativas de ocupaciones demandadas, de acuerdo con las capacidades de los trabajadores. Se estimaron dos regresiones multilogísticas, una para mujeres y otra para hombres.

El número de observaciones fue de 1153 (población de 132 604) para los hombres y de 1288 (población de 132 013) para las mujeres. En virtud de que la estimación se llevó a cabo con los ponderadores propuestos en la $\mathrm{ACS}^{10}$ fue posible evitar los problemas de heteroscedasticidad derivados de la heterogeneidad de los individuos (Kreuter y Valliant, 2007). Los resultados fueron satisfactorios en ambos modelos, los coeficientes en conjunto resultaron estadísticamente significativos en las dos regresiones de acuerdo con la prueba LR y con una pseudo R cuadrada de 0.12 para los hombres y de 0.16 para las mujeres. En otras palabras, el modelo con las variables explicativas respecto a un modelo con solo la constante es más adecuado en el caso de las mujeres.

En la Tabla 7 se presentan los coeficientes relativos ${ }^{11}$ de los dos modelos. Es decir, el cociente de la probabilidad de que ocurra un hecho o de que se elija una opción frente a la probabilidad de que no suceda el fenómeno o de que se elija la opción opuesta. Su interpretación es la "ventaja" o preferencia de una opción frente a otra, es decir, el número de veces que es más probable que ocurra el fenómeno frente a que no ocurra. Los comentarios de los resultados se presentan a continuación, organizados de acuerdo con los distintos tipos de variables: sociodemográficas, capital humano y condiciones migratorias.

\section{Características sociodemográficas}

Los grupos de edad resultaron significativos solo en el grupo masculino en edades 46 años y más de media calificación. Son los hombres mayores los que tienen menores oportunidades para incorporarse a trabajos que demandan, al menos, calificación mediana. A diferencia de ellos, la edad de las mujeres no parece tener relevancia.

La situación conyugal y la presencia de hijos sugieren una alta influencia en la condición laboral de las mujeres y no en los hombres. La condición de estar casada tiene un efecto negativo significativo en todos los niveles de calificación, al igual que tener descendencia, como se planteó en las hipótesis. Solo en el caso de los hombres, la situación conyugal de casado es significativa, pero al elevarse la probabilidad en las calificaciones medias.

\footnotetext{
${ }^{10}$ Es decir, mediante el comando svy en el software Stata.

${ }^{11}$ Conocidos en la literatura como razón de momios en español y odds ratio en inglés.
} 


\section{Capital humano}

Se comprueba la importancia del capital humano. Al tomar como referencia el grado de licenciatura, contar con posgrado incrementa las posibilidades de emplearse en puestos de alta calificación para los hombres y mujeres. Como era de esperarse, entre las mujeres en profesiones de calificación baja, contar con capital humano disminuye la probabilidad de emplearse en dichos puestos. Llama la atención que, para incorporarse a empleos que demandan calificaciones medias, no es significativo el capital humano, ni para hombres ni para mujeres.

Tabla 7: Factores asociados a la probabilidad de ocupar empleos de alta, media y baja calificación, 2015

\begin{tabular}{|c|c|c|c|c|c|c|}
\hline \multirow{2}{*}{ Características } & \multicolumn{2}{|c|}{ Alta } & \multicolumn{2}{|c|}{ Media } & \multicolumn{2}{|c|}{ Baja } \\
\hline & Hombres & Mujeres & Hombres & Mujeres & Hombres & Mujeres \\
\hline 36 a 45 & 1.41 & 1.27 & 1.10 & 1.69 & 1.26 & 1.26 \\
\hline Más de 46 & 0.35 & 1.62 & $0.25^{* *}$ & 1.74 & 0.43 & 1.40 \\
\hline Casado & 1.70 & $0.56^{* * *}$ & $1.84^{* *}$ & $0.61^{* *}$ & 1.01 & $0.53 * * *$ \\
\hline Hijos & 0.73 & $0.36^{* * *}$ & 0.94 & $0.28 * * *$ & 0.76 & $0.44^{* * *}$ \\
\hline Posgrado & $2.35^{* * *}$ & $1.80^{* * *}$ & 1.53 & 1.07 & 0.64 & $0.61 *$ \\
\hline ICMCC & 0.98 & 0.73 & 0.96 & 0.63 & 1.21 & $0.57 * *$ \\
\hline Ciencias sociales & 0.88 & 1.17 & 0.66 & 0.78 & 1.46 & 0.92 \\
\hline Edu-Salud & 0.98 & $0.62 * * *$ & 0.79 & $0.34^{* * *}$ & 1.00 & $0.60 * *$ \\
\hline Idioma & $4.31^{* * *}$ & $4.76^{* * *}$ & 1.84 & $3.94^{* * *}$ & 0.72 & 1.31 \\
\hline Ciudadanía & 1.34 & $2.57 * * *$ & 1.07 & $3.13^{* * *}$ & 0.65 & 1.24 \\
\hline Años residencia & $0.88^{* * *}$ & 0.96 & $0.90 * * *$ & 0.98 & $0.93^{* * *}$ & 0.99 \\
\hline Constante & $7.39 * * *$ & 0.55 & 8.34 & 0.31 & 33.03 & 2.17 \\
\hline
\end{tabular}

Fuente: Elaboración propia con base en Acs, 2015.

$\mathrm{p}^{* * *}<0.01 \mathrm{p}^{* *}<0.05 \mathrm{p}^{*}<0.10$

Nota: Mujeres: LR chi2(51) $=456.45$, Prob $>$ chi2 $=0.0000$, Pseudo R2 $=0.1601$

Hombres: LR chi2 $(51)=452.28$, Prob $>$ chi2 $=0.0000$, Pseudo R2 $=0.1292$

En relación con el campo de la formación académica, en la que se tomó negocios como referencia, la variable "otros" resultó estadísticamente significativa y menor a la unidad en ocupaciones de alta, media y baja calificación en el caso de las mujeres. Es decir, una formación de negocios posibilita que las mujeres pueden insertarse en ocupaciones de distintos niveles de calificación.

El dominio del inglés fue significativo para los empleos que demandan altas calificaciones tanto para hombres como para mujeres y en calificaciones medias solo para ellas, lo cual confirma la hipótesis planteada. 


\section{Condiciones migratorias}

Solo para las mujeres la ciudadanía resultó estadísticamente significativa en los empleos de calificaciones altas y medias. Mientras que entre los hombres no tiene un impacto significativo en ninguno de los casos. Estos resultados nos parecen sorprendentes.

El tiempo de residencia tiene un resultado no esperado ya que, tanto en los empleos que demandan altas calificaciones como medias, un año más de residencia disminuye la probabilidad de que sean ocupados por hombres. Algo similar ocurre con las mujeres en el caso de las ocupaciones de alta calificación.

Además de identificar cuáles son los principales factores explicativos de la inserción en las ocupaciones según grado de calificación y sus desigualdades por sexo, es interesante analizar las diferencias en la distribución de las probabilidades de ocuparse en las distintas opciones de mercado. Como se observa en la Figura 2, la probabilidad de que los hombres se empleen en ocupaciones de alta y media calificación es de $55 \%$ y la de las mujeres de $42 \%$. Por el contrario, la probabilidad de que las mujeres no trabajen o estén en empleos que demandan baja calificación es de $58 \%$ y de solo $45 \%$ para los hombres. Estas cifras reflejan las diferencias por sexo y la posición de desventaja que enfrentan las mujeres MP. Estos resultados son consistentes con los hallazgos de Gottfried (2013), quien señala que a pesar de que las migrantes han logrado grados universitarios y posgrados, y de que participan en una sociedad con marcados cambios en pro de la igualdad por sexo, la probabilidad de inserción laboral de las mujeres es significativamente menor a la de los hombres. Lo que hace difícil que compitan en términos de igualdad con los hombres fuera de la casa.

Figura 2: Probabilidad de emplearse en distintas ocupaciones por nivel de calificación, 2015

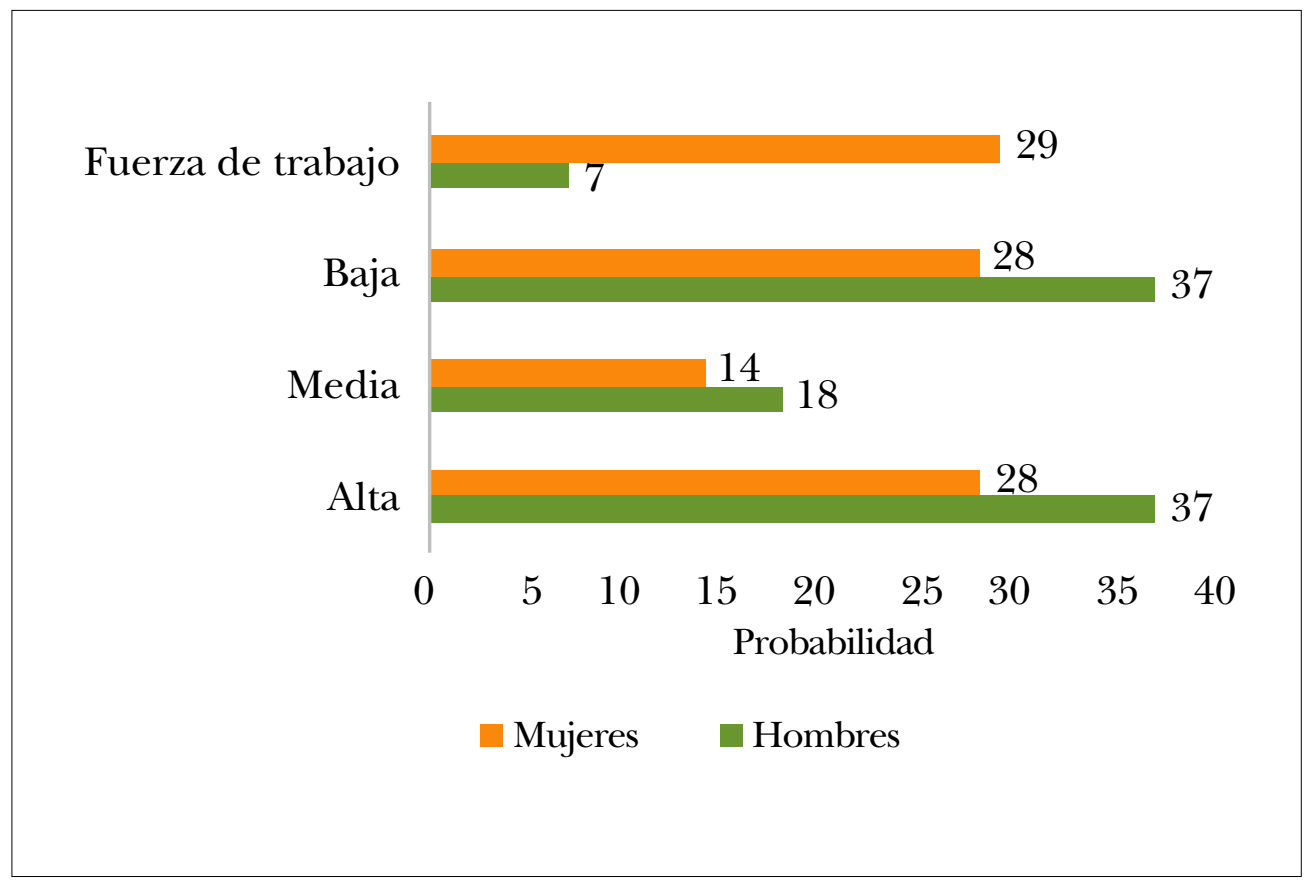

Fuente: Elaboración propia con base en Acs (2015). 


\section{La doble vulnerabilidad bajo distintos escenarios}

Dada la diversa influencia de las distintas variables por sexo, consideramos de interés identificar en qué tipo de ocupación se ubica la mayor probabilidad, en distintos escenarios: $i$ ) según la presencia de hijos y grado académico, ii) según condiciones migratorias, escolaridad y dominio del inglés.

En el primer escenario, se observa que los hombres tienen mayor probabilidad de emplearse en ocupaciones de calificación alta y en la de baja cuando su grado escolar es de licenciatura con o sin hijos. En las mujeres con un posgrado la mayor probabilidad corresponde a emplearse en ocupaciones de alta calificación cuando no hay hijos y estar fuera de la fuerza de trabajo frente a la presencia de hijos. Si cuentan solo con licenciatura y no tienen hijos, tienen mayor probabilidad de estar en ocupaciones de baja calificación y entre quienes tienen hijos, la probabilidad más alta también corresponde a estar fuera de la fuerza de trabajo. Cabe mencionar que la mayor probabilidad de las mujeres con hijos de participar en actividades fuera de la fuerza de trabajo puede sugerir que hay un patrón de género debido a la desigual carga de las labores reproductivas y de cuidado entre hombres y mujeres (Tabla 8). Como sugieren Benería y Roldán (1987) y Szasz (1994) el rol reproductivo socialmente asignado a las mujeres determina las posibilidades y características de su inserción laboral.

Tabla 8: Probabilidades de ocupaciones por tipo de calificación. Escolaridad e hijos, 2015

\begin{tabular}{ccccc}
\multicolumn{2}{c}{ Sin hijos } & & Con hijos \\
Escolaridad & Hombres & Mujeres & Hombres & Mujeres \\
\hline Licenciatura & Baja & Baja & Baja & Fuera de la fuerza trabajo \\
Posgrado & Alta & Alta & Alta & Fuera de la fuerza trabajo
\end{tabular}

Fuente: Elaboración propia con base en Acs (2015).

En el segundo escenario donde se relacionan las condiciones migratorias, la escolaridad y el dominio del idioma inglés con las probabilidades de ocuparse en distintos niveles de calificación, de la cual resultan cuatro combinaciones: contar con ciudadanía y dominio del inglés, solo el inglés, solo la ciudadanía y ninguna de las dos. La mejor combinación para hombres y mujeres es la primera, que da la mayor probabilidad de emplearse en ocupaciones de alta calificación, cualquiera que sea el grado académico. En la segunda combinación, donde se toma en cuenta el dominio del idioma, ocurre lo mismo para los hombres y las mujeres que cuentan con un posgrado. Con licenciatura, la mayor probabilidad se da en ocupaciones de baja calificación, tanto para hombres como para mujeres.

En caso de contar con ciudadanía y sin idioma, los hombres con posgrado tienen una alta probabilidad de desempeñarse en puestos de alta calificación. Por el contrario, las mujeres en este caso estarían probablemente fuera de la fuerza de trabajo. En este escenario, cuando se tiene licenciatura, tanto ellas como ellos se ubicarían con mayor probabilidad en empleos de baja calificación. 
Por último, en el peor escenario, de no contar con idioma ni ciudadanía, los hombres se emplearían en ocupaciones de baja calificación, tanto con licenciatura como con posgrado y las mujeres probablemente se ubiquen fuera de la fuerza de trabajo en ambos casos (Tabla 9). Así, las mujeres tienen mayor probabilidad de estar fuera de la fuerza de trabajo que los hombres, esto sugiere la presencia de desigualdades de género donde aún permea la mayor participación de las mujeres en la esfera privada. En el ámbito del hogar son ellas las encargadas del cuidado del hogar y los hijos, por lo que solo en presencia de las condiciones óptimas se rompe el patrón.

Estos resultados muestran cómo la vulnerabilidad como migrantes se intersecta con la vulnerabilidad de género, tal y como se menciona en la sección sobre migración y género.

Tabla 9: Probabilidades de ocupaciones por tipo de calificación. Escolaridad, idioma inglés y ciudadanía, 2015

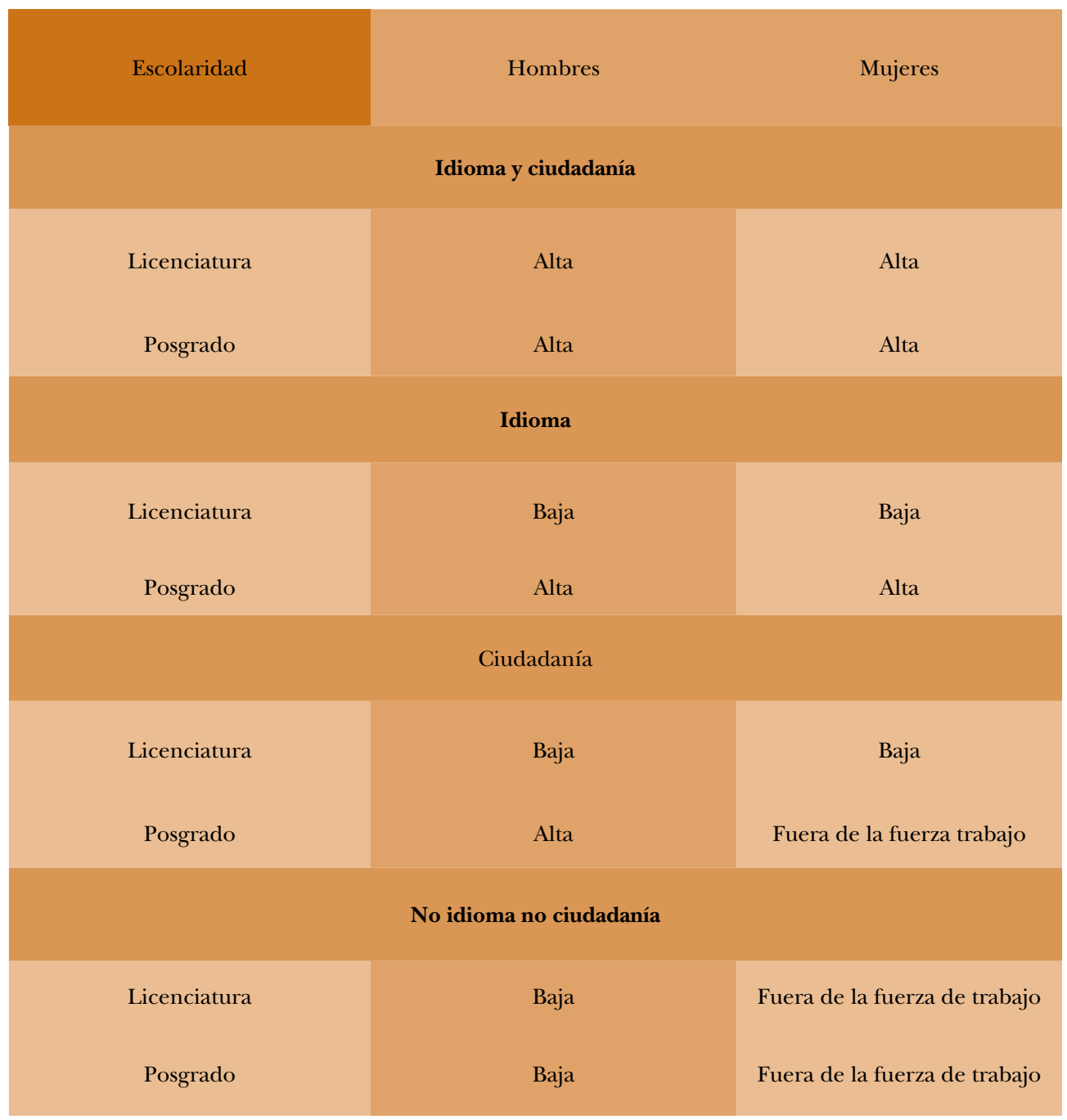

Fuente: Elaboración propia con base en Acs (2015). 


\section{Conclusiones}

En las últimas décadas se ha presentado un aumento de la migración calificada de mexicanos a Estados Unidos, en la cual las mujeres han tenido una participación creciente.

Con el fin de mostrar la presencia de un comportamiento muy diferenciado, este trabajo examina la inserción laboral por tipo de ocupación mediante la comparación entre hombres y mujeres.

Al interior del grupo de migrantes calificados existe segregación horizontal, manifiesta en una mayor concentración de las mujeres en ocupaciones identificadas como femeninas. Así mismo, la participación de las migrantes en ocupaciones de alta calificación destaca en aquellas relacionadas con educación y medicina. Mientras que en las de calificación media sobresale en servicios comunitarios y labores de oficina.

También está presente la segregación vertical, que da cuenta de una mayor aglutinación de las mujeres en puestos de menor jerarquía, a pesar de contar con niveles de calificación similares a sus pares varones (existencia y persistencia de techos de cristal). Así, en ocupaciones de gerencia, las migrantes calificadas tienen una menor presencia tanto en ocupaciones de calificación alta como media.

Por otra parte, los resultados de la estimación econométrica muestran que existen diferencias estadísticamente significativas entre los resultados de la regresión para mujeres y hombres. En relación con las características socioeconómicas, los grupos de edad solo resultaron significativos para los hombres en las ocupaciones de calificación alta y media, mientras que para las mujeres no tiene un efecto. Por el contrario, la situación conyugal y la presencia de hijos reducen la probabilidad de las migrantes calificadas de participar en ocupaciones de niveles alto, medio y bajo. Con respecto a las variables sobre capital humano, algunas especialidades de la formación académica fueron significativas solo para las mujeres: negocios, humanidades y ciencias sociales en calificación alta y, en calificación media: negocios, ICMCC y ciencias sociales. Mientras que para los hombres la única especialidad significativa fue ciencias de la salud en ocupaciones de calificación media. Cabe mencionar que los grados académicos, así como el dominio del inglés definitivamente incrementan la probabilidad de ocuparse en empleos de alta calificación, con alguna excepción. En lo que se refiere a las condiciones migratorias la ciudadanía solo es un factor determinante para las mujeres. En tanto que los años de residencia registraron un efecto negativo para la MP masculina y tienen signo positivo entre la MP femenina en ocupaciones de alta calificación.

Nuestros escenarios ilustran el patrón de género de la migración calificada. En el primer escenario que combina el grado académico con la presencia de hijos, fue contundente la alta probabilidad para la población femenina de estar fuera de la fuerza de trabajo, con la salvedad de contar con doctorado. En el segundo escenario - en el que se relacionan las condiciones migratorias, la escolaridad y el dominio del inglés-, se ilustra su vulnerabilidad como inmigrantes. Aquí se encontró que la ciudadanía es importante, pero el dominio del inglés lo es más y ante la dificultad de las mujeres de tomar cursos de idiomas y de capacitación cuando tienen hijos pequeños como señala Kofman (2012), es entendible que nuevamente resalten las desigualdades de género; en este caso las mujeres tienen mayor probabilidad de quedar fuera de la fuerza de trabajo que los hombres. 
Estos resultados coinciden con lo encontrado por varios autores (IOM-OECD, 2014; Kofman, 2012; Martínez, 2008; Ramírez-García y Gandini, 2016), quienes sugieren distintas causas. Martínez (2008) menciona la encrucijada entre la producción y la reproducción que persiste para las mexicanas en Estados Unidos. Kofman (2012) argumenta acerca de la imposibilidad para las mujeres inmigrantes de tomar cursos de idiomas, de capacitación y de certificación para desempeñarse profesionalmente en los países de llegada, sobre todo cuando tienen hijos pequeños y otras actividades familiares a su cargo. Es decir, los migrantes son vulnerables, pero la vulnerabilidad de género afecta doblemente a las mujeres. Por su parte, IOM-OECD (2014) señalan los sesgos en los procesos de admisión diseñados para atraer mano de obra calificada de otros países.

Es innegable que hay un grupo de mujeres que han roto las barreras de género en términos de los avances obtenidos en cuanto a formación académica y competencia en el mercado de trabajo, sin embargo, con frecuencia la carga de las obligaciones domésticas y de cuidados no remunerados — socialmente asignadas a ellas-, entre otros factores, pueden repercutir en su situación laboral. Para romper con esta inercia se requiere de políticas de conciliación con corresponsabilidad social, donde las tareas de trabajo doméstico y cuidados no remunerados sean compartidas entre mujeres y hombres, así como entre el Estado, el mercado y la sociedad. Se requiere de medidas y acciones que rompan con los mecanismos de invisibilidad que permean entre las mujeres.

\section{Referencias}

American Community Survey (ACS). (2015). Integrated Public Use Microdata Series: Version 7.0 [Base de datos]. Minneapolis, MN, Estados Unidos: University of Minnesota.

Batalova, J., Fix, M. y Creticos P. A. (2008). Uneven Progress: The Employment Pathways of Skilled Immigrants in the United States. Washington, DC: Migration Policy Institute.

Benería, L. y Roldán, M. (1987). The Crossroads of Class and Gender. Industrial Homework, Subcontracting, and Household Dynamics in Mexico City. Chicago: University of Chicago Press.

Caicedo, M. (2012). Participación económica de mujeres latinoamericanas y caribeñas en Estados Unidos. Gaceta Laboral, 18(1), 5-33. Recuperado de https:/ / produccioncientificaluz.org/index.php/gaceta/article/view/3822

Cárdenas, M. (1983). La función social de las esposas de los migrantes a los Estados Unidos: el caso de Chavinda, Michoacán, Ponencia presentada en el Primer Foro Regional sobre Investigación y Cambio Social en Michoacán, Zamora, Michoacán.

Cerrutti, M. y Massey, D. (2004). Trends in Mexican Migration to the United States, 1965-1995. En J. Durand y D. Massey (Eds.), Crossing the Border: Research from the Mexican Migration Project (pp. 17-44). Nueva York: Russell Sage Foundation.

Clasificación Internacional Uniforme de Ocupaciones (CIUO). (2008). Organización Internacional del Trabajo. Ginebra: OIT.

Delgado, R. (2015). La migración mexicana altamente calificada: elementos para una política nacional en ciencia y tecnología. México: Conacyt-unesco. 
Delgado, R., Chávez, M. y Rodríguez, H. (2016). La innovación y la migración calificada en la encrucijada: reflexiones a partir de la experiencia mexicana. Revista Interdisciplinaria de Movilidad Humana, 24(47), 153-174. Recuperado de https:/ / www.redalyc.org/pdf/4070/407047127010.pdf

Docquier, F., Lowell, B. L. y Marfouk, A. (2009). A Gendered Assessment of Highly Skilled Emigration. Population and Development Review, 35(2), 297-321. doi: https://doi.org/10.1111/j.1728-4457.2009.00277.x

Domínguez, L. y Brown, F. (2013). Diferencias de género en la elección del sitio de trabajo en un contexto de crisis. Revista CEPAL, (111), 83-102.

Elson, D. (1999). Labor Markets as Gendered Institutions: Equality, Efficiency and Empowerment Issues. World Development, 27(3), 611-627. https://doi.org/10.1016/ S0305-750X (98)00147-8

Eurostat. (2010). EU Labour Force Survey [Base de datos]. Luxembourg: Eurostat.

Feixa, C. (2008). Generación uno punto cinco. Revista de Estudios de Juventud, (80), 115-127. Recuperado de http://www.injuve.es/sites/default/files/2012/44/ publicaciones/revista-80-capitulo-7.pdf

Feliciano, C. (2008). Gendered Selectivity: U.S. Mexican Immigrants and Mexican Nonmigrants, 1960-2000. Latin American Research Review, 43(1), 139-160. doi: https://doi.org/10.1353/lar.2008.0009

Gandini, L. (2019). Explicaciones de la migración calificada: el papel de las mujeres desde la experiencia norteamericana. Estereotipos, sesgos y desafíos. Norteamérica, 14(1), 369-390. doi: http://dx.doi.org/10.22201/cisan.24487228e.2019.1.371

García, B. y Pacheco, E. (2000). Esposas, hijos e hijas en el mercado de trabajo de la Ciudad de México en 1995. Estudios Demográficos y Urbanos, 15(1), 35-63. doi: http://dx.doi.org/10.24201/edu.v15i1.1066

Gaspar, S. y Chávez, M. (2016). Migración mexicana altamente calificada: 19902013. Problemas del Desarrollo, 47(185), 81-110. doi: https://doi.org/10.1016/j. rpd.2016.04.002

González, J. G. (2005). Inserción laboral de los migrantes calificados de origen mexicano en Estados Unidos, 1990-2000. Revista Argentina de Sociología, 3(5), 88-106. Recuperado de http://dialnet.unirioja.es/servlet/oaiart?codigo $=1420466$

Gottfried, H. (2013). Gender, Work, and Economy. Unpacking the Global Economy. Cambridge: Polity Press.

International Organization for Migration (Iом). (2010). Migration and Gender. Geneva: IOM.

IOM-OECD. (2014). Harnessing Knowledge on the Migration of Highly Skilled Women. Ginebra: IOм. Recuperado de https://publications.iom.int/es/books/harnessing-knowledge-migration-highly-skilled-women

Kanaiaupuni, S. M. (2000). Reframing the Migration Question: An Analysis of Men, Women, and Gender in Mexico. Social Forces, 78(4), 1311-1347. doi: $10.2307 / 3006176$

Kofman, E. (2012). Gender and Skilled Migration in Europe. Cuadernos de Relaciones Laborales, 30(1), 63-89.

Kreuter, F. y Valliant, R. (2007). A Survey on Survey Statistics: What is done and Can Be Done in Stata. The Stata Journal, 7(1) 1-21. Recuperado de https://www.stata-journal.com/article.html?article=st0118 
Lowell, L. y Pederzini, C. (2012). Gender Differentials in Emigration by Level of Education: Mexican-Born Adult Migrants in the United States. En C. Pederzini y A. Cuecuecha (Eds.), Migration and Remittances From Mexico. Trends, Impacts and New Challenges (pp. 159-184). Reino Unido: Lexington Books.

Man, G. (2004). Gender, Work and Migration: Deskilling Chinese Immigrant Women in Canada. Women's Studies International Forum, 27(2), 135-148. https://doi.org/10.1016/j.wsif.2004.06.004

Martínez, J. (2008). La migración calificada en América Latina: viejos, persistentes y nuevos temas. Seminario Internacional Diásporas y Circulación de Talentos, ¿̇Una movilidad al servicio del desarrollo en América Latina? Santiago de Chile: CELADE-CEPAL.

Meares, C. (2010). A fine balance: Women, Work and Skilled Migration. Women's Studies International Forum, 33(5), 473-481. doi: https://doi.org/10.1016/j. wsif.2010.06.001

OECD. (2016). Social Expenditure Database. París: OECD.

oECD. (2017). G20 Global Displacement and Migration Trends Report 2017. París: oEcD.

Orozco, A. (2007). Documento de trabajo 2: Cadenas globales de cuidado. República Dominicana: INSTRAW.

Perrons, D. (2015). Gendering the Inequality Debate. Gender E Development, 23(2), 207-222. doi: https://doi.org/10.1080/13552074.2015.1053217

Ramírez-García, T. y Gandini, L. (2016). Trabajadoras calificadas: las mujeres mexicanas en el mercado de trabajo estadounidense en perspectiva comparada. Revista Latinoamericana de Población, 10(19), 33-56. Recuperado de http://www.revistarelap.org/index.php/relap/article/view/48/68

Ramírez-García, T. y Tigau, C. (2018). Mujeres mexicanas altamente calificadas en el mercado laboral estadunidense: cintegradas o segregadas? Sociedad y Economía, 34, 75-101.

Rodríguez, C. (2012). La cuestión del cuidado: ¿El eslabón perdido del análisis económico? Revista CEPAL, (106), 23-36. Recuperado de https://www.cepal.org/es/ publicaciones/11524-la-cuestion-cuidado-eslabon-perdido-analisis-economico

Rodríguez, M. M. (2014). Las mujeres migrantes como protagonistas de la migración calificada en América Latina y el Caribe. En P. Galeana (Coord.), Historia comparada de las migraciones en las Américas (pp. 421-438). México: Universidad Nacional Autónoma de México.

Stone, P. (1994). Assessing Gender at Work: Evidence and Issues. En J. Jacobs (Ed.), Gender Inequality at Work (pp. 408-423). California: Sage.

Szasz, I. (1994). Migración y relaciones sociales de género: aportes desde la perspectiva antropológica. Estudios Demográficos y Urbanos, 9(1), 129-150. doi: http:// dx.doi.org/10.24201/edu.v9i1.903

Vázquez, L. y Domínguez, L. (2018). La inserción laboral de migración contemporánea de mexicanos profesionistas a EU: un análisis comparativo. Sociedad y Economía, (34), 27-49. doi: https://doi.org/10.25100/sye.v0i34.6460

Walby, S., Gottfried, H., Gottschall, K. y Osawa, M. (Eds.). (2016). Gendering the Knowledge Economy Comparative Perspectives. Londres: Palgrave.

Zhou, M. (2003). Contemporary Trends in Immigration to the United States: Gender, Labor-Market incorporation, and Implications for Family Formation. Migraciones Internacionales, 5(2), 76-96. Recuperado de https://migracionesinternacionales.colef.mx/index.php/migracionesinternacionales/issue/view/80 
Lilia Domínguez Villalobos

Mexicana. Doctora en Ciencias Económicas por la Universidad Autónoma Metropolitana. Maestra en Economía en la Universidad de East Anglia, Inglaterra y Licenciada en Economía por la Universidad Nacional Autónoma de México. Profesorainvestigadora en el posgrado de la Facultad de Economía de la unAM. Miembro del Sistema Nacional de Investigadores nivel III. Líneas de investigación: organización industrial, medio ambiente, género y migración. Publicación reciente: Ríos, E. I. y Domínguez, L. (2018). Patrones de competencia y su efecto en los precios de los medicamentos de patente expirada en México: el caso de los antibióticos (2014-2015). Economía, XLI(82), 126-156.

\section{Flor Brown Grossman}

Mexicana. Doctora en Ciencias Económicas por la Universidad Autónoma Metropolitana. Maestra en Economía por la Maestría en Docencia Económica de la Universidad Nacional Autónoma de México y Licenciada por la Universidad Autónoma Metropolitana. Profesora-investigadora en la Universidad Autónoma de Querétaro. Miembro del Sistema Nacional de Investigadores nivel III. Líneas de investigación: organización industrial, cambio técnico y productividad. Publicación reciente: Nava, I. y Brown, F. (2018). Determinantes del ahorro de los hogares en México: un análisis de regresión cuantílica. Economía Teoría y Práctica, (49), 93-118.

\section{Isalia Nava Bolaños}

Mexicana. Doctora en Estudios de Población y Maestra en Demografía, ambas por El Colegio de México. Licenciada en Economía, por la Universidad Nacional Autónoma de México. Investigadora del Instituto de Investigaciones Económicas de la UnAM. Miembro del Sistema Nacional de Investigadores nivel I. En 2012 obtuvo el primer lugar del Premio Gustavo Cabrera Acevedo otorgado por El Colegio de México, en la modalidad de investigación en población. Obtuvo el reconocimiento Distinción Universidad Nacional para Jóvenes Académicos 2017, en el área de investigación en ciencias económicas-administrativas. Líneas de investigación: demografía y economía, impacto económico del cambio poblacional, economía y envejecimiento de la población, género y economía. Publicación reciente: Nava, I. y Brown, F. (2018). Determinantes del ahorro de los hogares en México: un análisis de regresión cuantílica. Economía Teoría y Práctica, (49), 93-118. 